\title{
Uso de juegos digitales educativos como herramienta de soporte para el aprendizaje de algoritmos
}

\section{Use of educational digital games as support tool for algorithms learning}

\author{
Julián Moreno, Universidad Nacional de Colombia, UFRGS \\ jmoreno1@unal.edu.co
}

\begin{abstract}
Resumen
Como lo mencionan muchos autores, el aprendizaje de algoritmos y programación es una tarea difícil no sólo por el alto contenido lógico y abstracto que representa, sino también porque en muchas ocasiones los procesos de enseñanza no cuentan con métodos y herramientas que permitan "llegar" de forma efectiva a los estudiantes. Buscando una solución a esta situación, en este trabajo se propone el uso de juegos digitales los cuales por medio de un diseño bien definido, permiten que los estudiantes asimilen ciertos conceptos como secuenciación, iteración definida, llamado de funciones y anidamiento de instrucciones; al tiempo que mejoran sus habilidades para usarlos en la resolución de problemas.
\end{abstract}

Palabras clave: juego digital educativo, algoritmo, programación

\begin{abstract}
As has been mentioned by a lot of authors, the learning of algorithms and programming is a difficult task not only because the high logic and abstract content that it represents, but also because in many times the teaching processes do not count with methods and tools that allow reaching the students in an effective way. Looking for a solution to this situation, this work proposes the use of digital games which, with a well defined design, allow students assimilating certain concepts like sequencing, defined iteration, functions calling and instructions nesting; and at the same time improving the ability of using them in the resolution of problems.
\end{abstract}

Keywords: educational digital game, algorithm, programming

\section{Introducción}

Una amplia variedad de estudios señalan la relevancia de los juegos educativos para alcanzar diversos objetivos de aprendizaje bien sea de tipo verbal, matemático, lógico, visual, motor-sensorial o de resolución de problemas (Klopfer \& Yoon, 2005). Pero ¿cómo se logra esto?, ¿cómo los juegos digitales en particular pueden ayudar en los procesos cognitivos? Piaget (1983) analiza la importancia de la relación entre sujeto y objeto (en este caso el juego) y como las reacciones del segundo a las acciones del primero le permiten a este construir modelos mentales los cuales, por medio del proceso de interacción, ganan significancia y se convierten en una parte fundamental del proceso de aprendizaje.

Como se menciona en (James et al., 2003) diversos estudios muestran que los juegos digitales pueden aumentar diálogos significativos entre los estudiantes y que 
tienen efectos positivos en las habilidades sociales. Este mismo autor argumenta que utilizar estos recursos para el aprendizaje abre enormes oportunidades para llevar experiencias de la vida cotidiana a las aulas de clase, aumentando la motivación y el compromiso con el aprendizaje. Para reforzar esta idea, en (Lewandowski \& Soares, 2008) se menciona que los juegos pueden ser una herramienta auxiliar del alumno en la construcción de su conocimiento sistematizado. Esta sistematización posibilita un mejor acompañamiento del alumno verificando sus errores más frecuentes y presentándole recursos de manera llamativa, diferente a como se hace en el modo convencional.

En el área específica del aprendizaje de computación e informática existen diversos trabajos que exploran el uso de juegos digitales educativos en varios campos tales como: conceptos básicos de computación (Gorriz \& Medina, 2000), sistemas operativos (Hill et al., 2003), estructuras de datos (Lawrence, 2006), ingeniería de software (Baker et al., 2005; Zapata, 2009), y algoritmos y programación (Moser, 1997; Becker, 2001; Rapkiewickz, 2006). Este último es precisamente el objeto de estudio de este trabajo siendo de particular interés dados los desafíos que representa tanto para profesores como para estudiantes, los cuales muchas llevan a problemas graves como la desmotivación seguida de reprobación o evasión.

Como se menciona en (Rodriguez, 2002) se destacan entre estos desafíos la dificultad de los estudiantes para desarrollar razonamiento lógico principalmente cuando están acostumbrados a "llenar" contenidos, así como el desanimo que muchos pueden sentir al creer que la disciplina constituye un obstáculo extremamente difícil de superar. En adición a esto, Pereira \& Rapkiewickz (2000) apuntan que gran parte de los estudiantes no consiguen asimilar las abstracciones y conceptos necesarios, imposibilitando así el posterior desarrollo de programas.

Considerando este panorama, así como la importancia de esta disciplina, tanto para programas técnicos como profesionales, en este artículo se describe el uso de unos juegos digitales educativos desarrollados en la Universidad Nacional de Colombia Sede Medellín, para apoyar el aprendizaje y mejorar las habilidades en la utilización de algunos conceptos básicos como son la secuenciación de instrucciones, la iteración definida, los procesos anidados y el llamado de funciones.

El resto de este artículo está organizado como se describe a continuación. En la sección 2 se presenta el marco conceptual sobre el cual se fundamenta este trabajo, es decir, el aprendizaje basado en juegos: En la sección 3 se describen los principios seguidos para el diseño de los juegos desarrollados, mientras que en la sección 4 se presentan y analizan algunos resultados preliminares. Por último en la sección 5 se finaliza mencionando algunas conclusiones, así como el trabajo futuro.

\section{Aprendizaje basado en juegos}

El aprendizaje basado en juegos o GBL (por sus siglas en inglés de "Game Based Learning) puede considerarse un área de investigación relativamente nueva cuyo objeto de estudio son los juegos con capacidad de balancear un dominio de conocimiento o habilidad cognitiva específica con la capacidad del aprendiz en su rol de jugador para retenerlos y aplicarlos como parte de su proceso de aprendizaje y posteriormente en situaciones de la vida real. Para alcanzar este objetivo, el éxito de las estrategias basadas en GBL radica en dos aspectos. El primero es que la interacción activa es el centro de la experiencia y el segundo es que el aspecto motivacional es un elemento fundamental de este enfoque. Como lo menciona (Gómez et al., 2004), un 
juego puede mostrar que tareas difíciles y tediosas pueden ser entretenidas cuando forman parte de una buena historia.

En (Robertson \& Howells, 2008) se describe un ciclo de cuatro partes donde el jugador analiza y prueba, crea hipótesis, reprueba y repiensa; estableciendo así un enlace entre sus acciones y la respuesta del juego, con el cual consigue replantear sus modelos mentales. Un ciclo similar es definido por (Garris et al., 2002), como se muestra en la figura 1, en el que el estudiante son su rol de jugador lleva a cabo un ciclo de juicio - comportamiento - realimentación, que le permite traducir tanto las características del juego como el contenido instruccional incorporado en un nuevo conocimiento o en la mejora de sus habilidades.

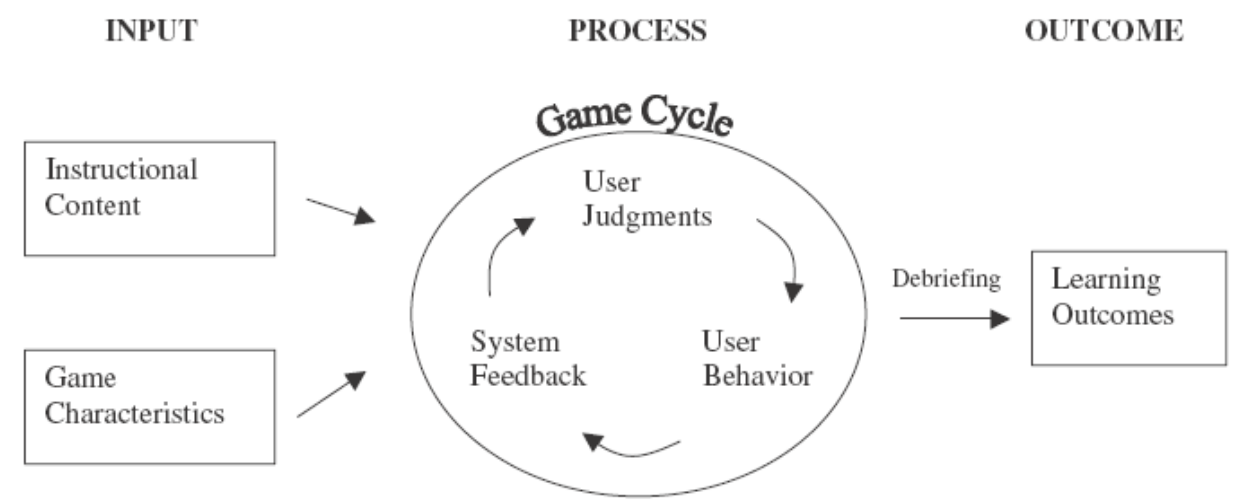

Figura 1. Modelo entrada-procesamiento-salida (Garris et al., 2002)

Para lograr este ciclo y permitir un aprendizaje efectivo, este mismo autor analiza los factores que caracterizan los juegos y define seis grandes dimensiones como se presenta a continuación.

- Fantasía: desplegar un contexto imaginario tanto de ambiente como de personajes.

- Reglas y objetivos: ambos deben ser claramente definidos para que el jugador entienda su progreso, recibiendo la realimentación adecuada.

- Estímulos sensoriales: presentar estímulos visuales y auditivos que acompañen el "drama" del contexto del juego.

- Desafíos: contener una dificultad adecuada que incentive al jugador a avanzar.

- Misterio: definir cierto nivel de complejidad y formato en la información que se le presenta al estudiante para mantenerlo interesado.

- Control: permitir un control activo del jugador.

\section{Principio de diseño de los juegos}

A partir del panorama mencionado en la sección 1 así como de las consideraciones generales descritas en la sección 2, se desarrollaron una serie de juegos educativos para el área específica de algoritmos y programación siguiendo los principios explicados a continuación.

a) Ser altamente interactivos: la idea general del juego es que el estudiante controla un robot llamado ProBot (Programmable Robot) para participar en deportes como tenis y boxeo, y cuyos movimientos son determinados a partir de 
algoritmos que el estudiante debe definir. Bajo este concepto toda acción ejecutada por el protagonista del juego nace de la interacción directa del jugador. Para dar un ejemplo, en la figura 2 se muestra un algoritmo para el juego de boxeo. En la parte izquierda se muestran las instrucciones que pueden emplearse y en la derecha la secuencia definida por el estudiante. En este ejemplo, de las instrucciones disponibles se define repetir tres veces, por medio de una estructura de iteración definida, la secuencia bloquear golpe a la cabeza y dar golpe al cuerpo.

Instrucciones disponibles
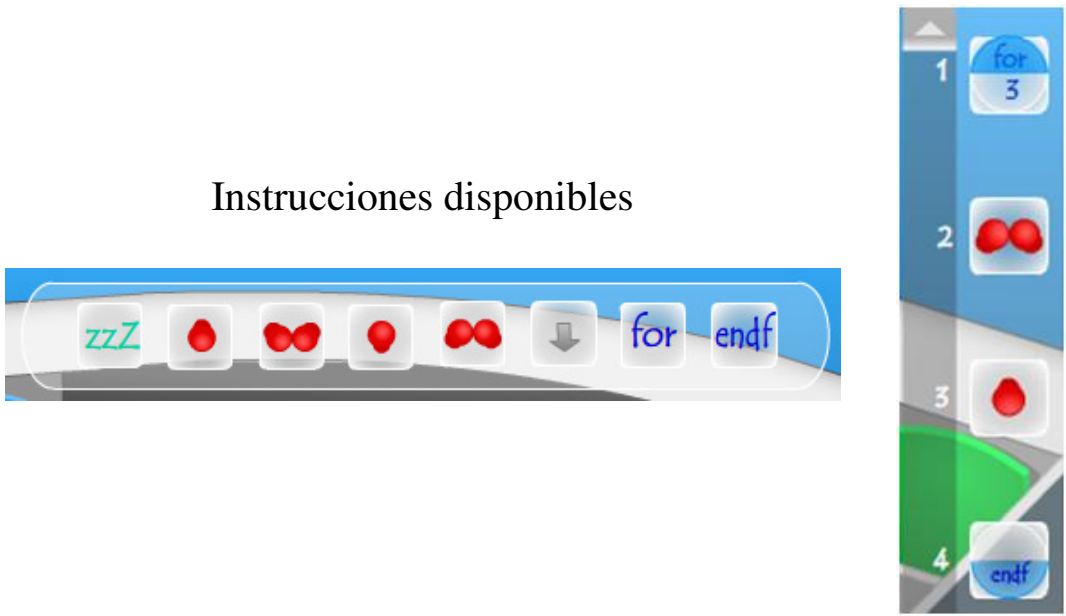

Algoritmo

\section{Figura 2. Ejemplo de definición de algoritmos}

b) Tener una relación directa con los conceptos del programa curricular de interés: al definir los movimientos que el protagonista tiene que realizar para ganar en las contiendas, el jugador internamente se está apropiando de los conceptos de la disciplina tales como secuenciación de instrucciones, iteración definida, procesos anidados y llamado de funciones. Los juegos están diseñados de tal manera que se imposibilitan las victorias "al azar", es decir, que el estudiante necesariamente tiene que reflexionar sobre tales conceptos para lograr avanzar.

c) Tener reglas bien definidas: los juegos funcionan a manera de compilador, es decir, se encargan de traducir los algoritmos definidos por el jugador y expresados por medio de "instrucciones" en los movimientos del protagonista. En este sentido, si bien la escritura de tales algoritmos es libre, está reglamentada por las instrucciones disponibles. En palabras más simples, el jugador puede hacer lo que se le ocurra para ganar las disputas, empleando solamente las instrucciones disponibles.

d) Ser intuitivos: los juegos deben ser fáciles de usar y con controles amigables. Para esto las interfaces cuentan con instrucciones simples que los jugadores pueden arrastrar y soltar para crear sus algoritmos. Adicional a esto, cuando los jugadores prueban sus soluciones, la interfaz exhibe una ejecución paso por paso que les permite en todo momento verificar su ejecución.

e) Ser visual y auditivamente atractivo pero sin contener carga cognitiva extra: En las figuras 3 y 4 puede verse que si bien las interfaces de los juegos son 
relativamente simples desde el punto de vista de la multimedia, logran ser estéticamente llamativos, pero sin tener demasiados efectos distractores que acaparen la atención de los estudiantes.

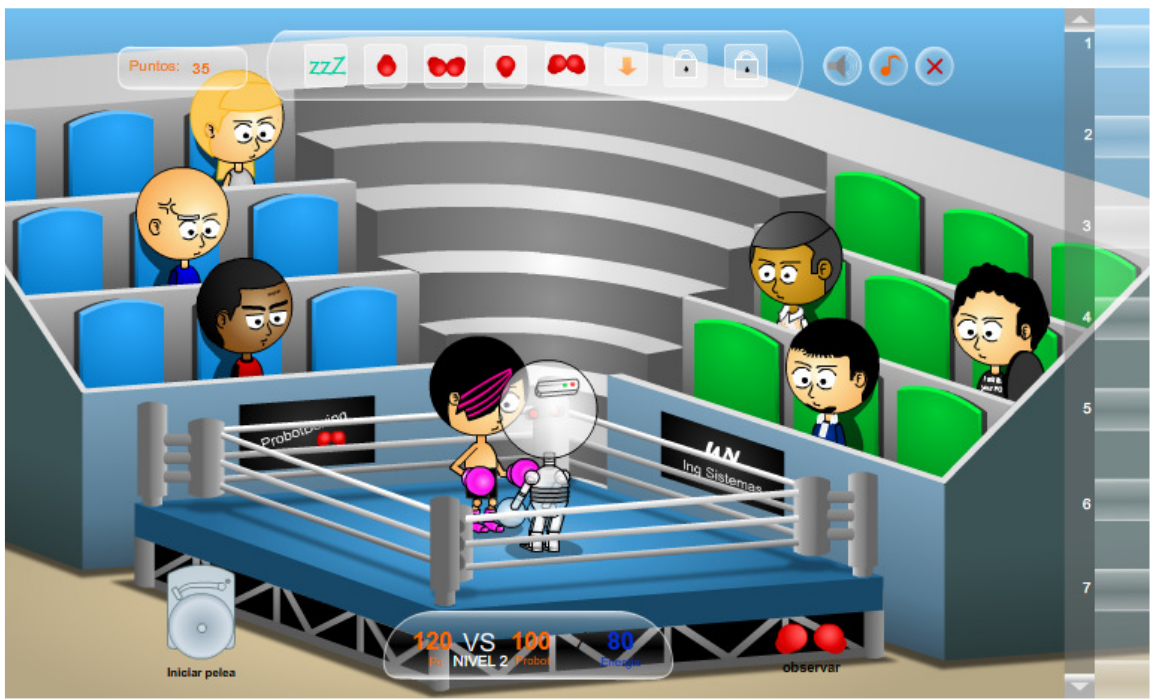

Figura 3. Interface de juego para el aprendizaje de iteración definida

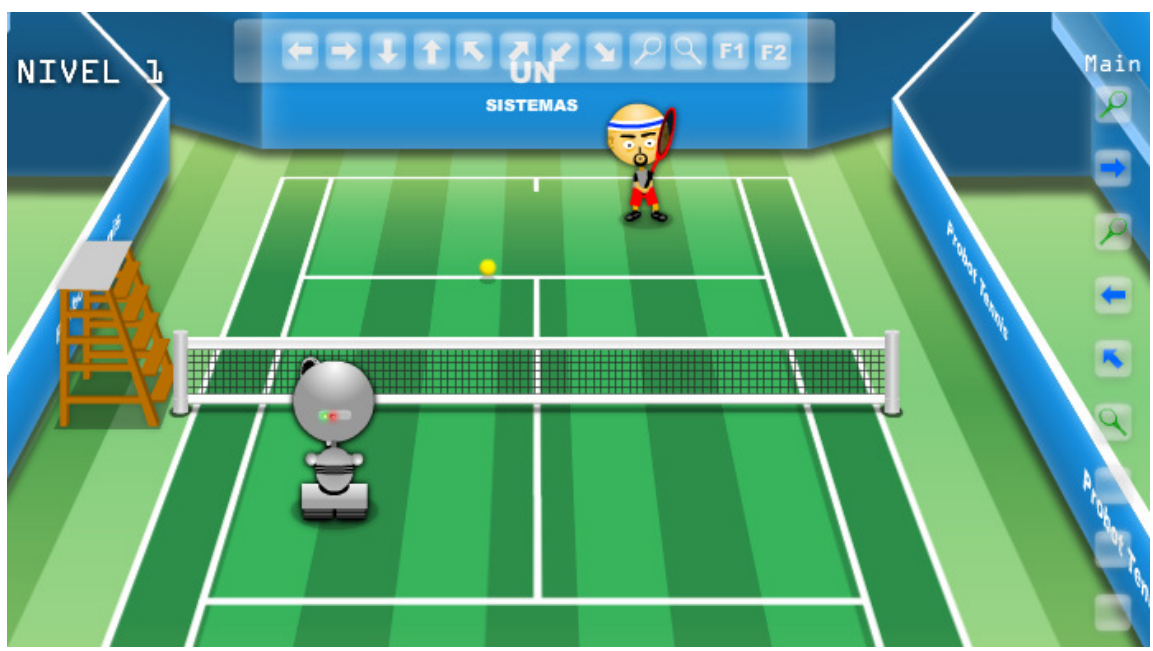

Figura 4. Interface de juego para el aprendizaje de llamado a funciones

f) Poseer una realimentación inmediata e ilimitada: tal realimentación se presenta un dos niveles, uno a nivel de la ejecución de los algoritmos planteados para que los estudiantes puedan visualizar las consecuencias de las decisiones tomadas y alteren así sus modelos mentales; y otra a nivel de su finalización, donde los estudiantes descubren si sus posibles soluciones resuelven o no el problema. 


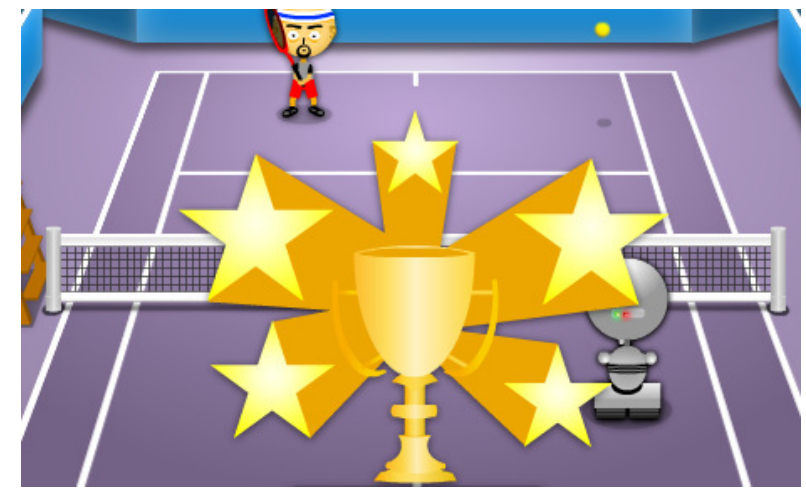

Figura 5. Ejemplo de mensaje de victoria

En caso afirmativo el juego presenta imágenes de éxito como la presentada en la figura $5 \mathrm{y}$ en caso contrario le permite intentar de nuevo, bien sea que el jugador modifique la solución o proponga otra completamente diferente.

g) Ser estimulantes: esto se logra por una parte partiendo de una aproximación de resolución de problemas y por otra agregando diversos niveles de dificultad a medida que el jugador avanza. Tal progreso incremental además de ser el mecanismo común de los juegos para mantener el interés del jugador, permite un abordaje natural a los diferentes conceptos que se desean analizar. En otras palabras se comienza por los conceptos más simples, a los que gradualmente se van incorporando otros, hasta lograr una comprensión no sólo general sino discriminante de todos.

h) Detectar problemas: los juegos proveen mecanismos de alerta a la hora de detectar errores tanto simples como de comprensión de conceptos por parte de los estudiantes. En el contexto de los algoritmos un error simple puede ser por ejemplo un error sintáctico o semántico donde no se afecta la lógica como tal del algoritmo sino que simplemente está "mal escrito".

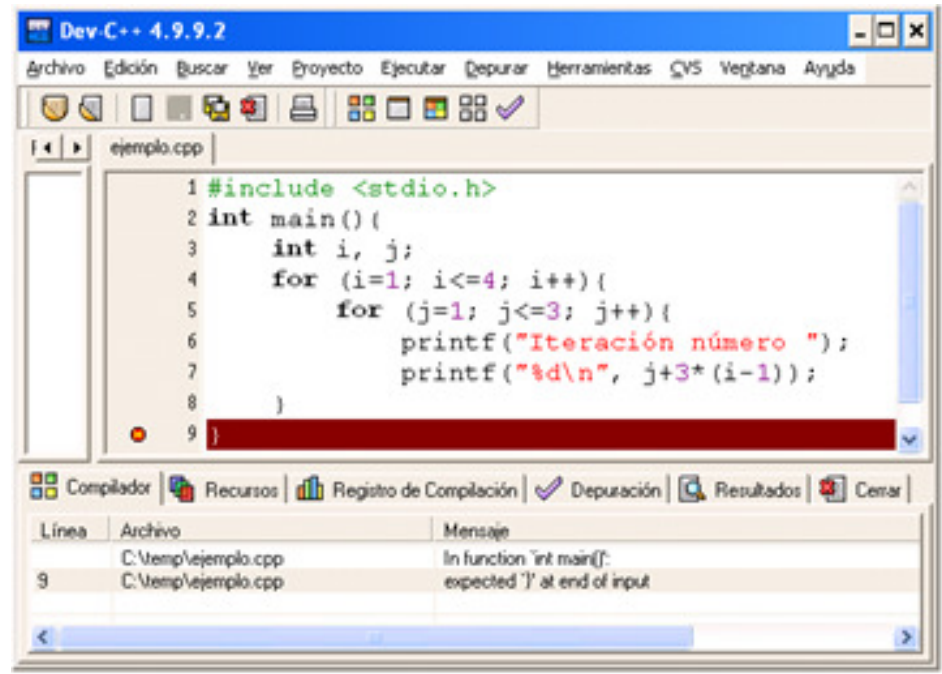

Figura 6. Ejemplo de error semántico 


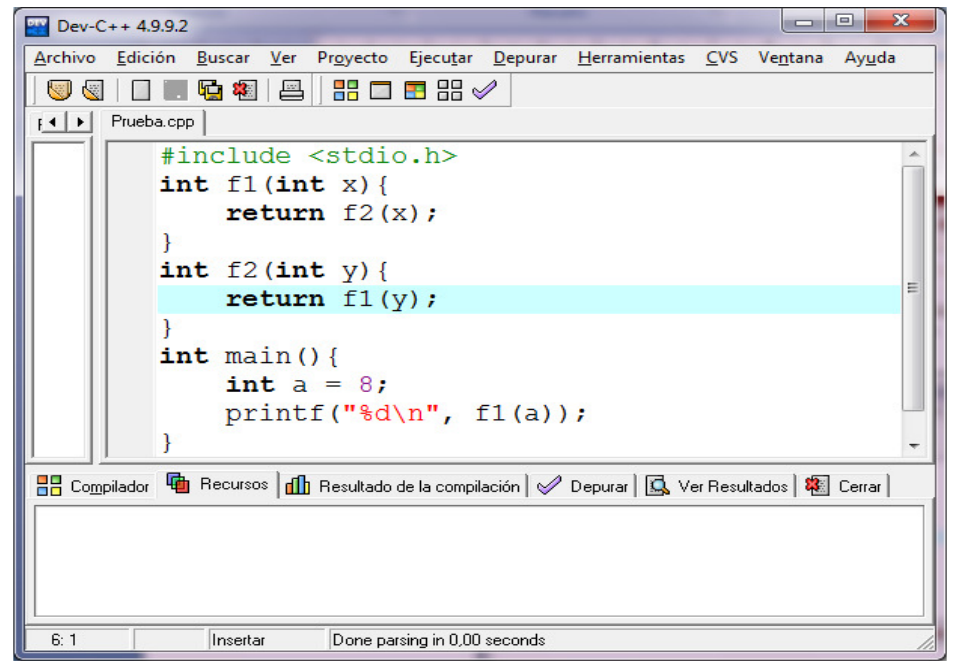

Figura 7. Ejemplo de error lógico

Un ejemplo de este tipo de error, no dentro del juego si no en un código real, se muestra en la figura 6: no cerrar una instrucción de iteración. Por otro lado un error de comprensión, indica un problema en el entendimiento de algún concepto, evidenciado en un error lógico dentro del algoritmo. Un ejemplo de este tipo de error, nuevamente para un código real, se presenta en la figura 7: una recursión infinita.

i) Contar con mecanismos que promuevan el mejoramiento continuo: al ganar un nivel se presenta el puntaje obtenido, el cual es directamente proporcional a la eficiencia de la solución propuesta en términos de economía de recursos. Esta eficiencia hace referencia a aspectos como el uso de recursos disponibles ("energía" y "sangre" por ejemplo), cantidad de líneas de código, etc. Adicional a esta validación "individual" que el estudiante puede hacer, un aspecto fundamental de los juegos es que al hacer una comparación de los puntajes de otros jugadores es posible determinar si alguien encontró o no soluciones mejores. En una prueba piloto se contó por ejemplo con un cuadro de puntuación como el mostrado en la tabla 1 el cual, como los mismos estudiantes manifestaron, fue un factor determinante en la motivación. Un fenómeno observado es que varios estudiantes pese a haber completado un nivel, muchas veces lo repetían buscando nuevas soluciones para mejorar su puntuación, replanteando en este proceso sus modelos mentales y consecuentemente mejorando sus habilidades.

Tabla 1. Ejemplo de cuadro de puntuación

\begin{tabular}{ccc}
\hline Nombre & Nivel & Puntuación \\
\hline- & 5 & 255 \\
- & 5 & 225 \\
- & 4 & 210 \\
- & 3 & 205 \\
- & 4 & 185 \\
- & 3 & 120 \\
- & 3 & 105 \\
- & 2 & 90 \\
- & 2 & 70 \\
\hline
\end{tabular}




\begin{tabular}{lll}
\hline- & 2 & 45 \\
- & 1 & 45 \\
- & 1 & 35 \\
- & 2 & 25 \\
\hline
\end{tabular}

j) Ser accesibles: los juegos fueron implementados en Macromedia Flash con el fin que los estudiantes puedan acceder a él desde un navegador Web, lo cual facilita enormemente su acceso pues si bien no el $100 \%$ de ellos cuentan con un computador y mucho menos Internet en casa, todos si tienen la posibilidad de ingresar a las diversas salas de cómputo con las que cuenta la Universidad.

\section{Resultados preliminares}

Con el fin de validar los juegos desarrollados se llevó a cabo una prueba piloto en la que se permitió a un grupo de cerca de 60 estudiantes de diferentes semestres de programas de ingeniería interactuar con ellos tanto de manera libre como en competencia. Luego de dicha interacción, la cual fue monitoreada para registrar los comportamientos e impresiones, se llevó a cabo una encuesta escrita con cuatro puntos principales para determinar: 1) cual fue la apreciación general de los juegos, 2) cual era la opinión respecto a su utilización como material educativo, 3) que pensaban sobre su atractivo multimedia, es decir, respecto a gráficos, animaciones, sonidos, etc. 4) que opinaban sobre los asuntos de usabilidad, es decir si los controles y las reglas eran fáciles de entender y utilizar. Luego de que los estudiantes diligenciaron la encuesta de manera anónima (para evitar que se sintieran presionados) usando una escala cuantitativa de uno a diez para cada punto, se obtuvieron los siguientes valores promedio respectivamente: $8.12,8.56,8.67$ y 7.67 , con unas desviaciones estándar correspondientes de $1.41,1.57,1.33$ y 2.25 , los cuales demuestran una apreciación general de los estudiantes muy positiva.

Después de responder esos cuatro puntos de manera cuantitativa se les permitió a los estudiantes escribir libremente sobre su experiencia y, de los comentarios recibidos, tres tipos fueron detectados: respecto al uso del juego en aulas de clase, respecto a su comparación con otros juegos y respecto al aspecto de la competencia. En el primer caso "Es bueno aprender jugando" y "Me gustaría estudiar haciendo algo entretenido como en el juego", fueron por ejemplo algunos de los comentarios recibidos, demostrando así el efecto motivacional que este tipo de recursos puede aportar en los procesos de aprendizaje. En el segundo caso la mayoría de los comentarios estaban relacionados con el hecho que pese a que muchos de los estudiantes están acostumbrados a juegos más sofisticados en cuanto a gráficos, sonidos, efectos y narrativa de las consolas comerciales actuales, estos encontraron los juegos agradables y muy divertidos. Si bien divertir este no es el objetivo principal de los juegos educacionales, si se convierte en un elemento importante que sin duda alguna ayuda a capturar el interés de los estudiantes lo cual, en el caso de estudiantes en edades de 16 a 20 años que fue el caso de la prueba piloto, como bien lo saben los profesores, no es una tarea fácil. En el último caso muchos comentarios apuntaron a la importancia de contar con mecanismos de competencia como es el caso de los cuadros de puntuación, pues estos alientan a los estudiantes de manera sana a esforzarse y mejorar sus habilidades. "Cada vez que terminaba un nivel, me fijaba en la tabla de 
puntuaciones para ver que tan bien estaba comparado con los otros", dijo uno de los estudiantes.

\section{Conclusiones}

Considerando la importancia que tienen las disciplinas relacionadas con algoritmos y programación dentro de numerosos programas de formación, pero en especial los desafíos que supone su aprendizaje, en este artículo se describió el uso de juegos digitales educativos cuyo objetivo es servir de apoyo en este proceso, haciendo hincapié en los principios seguidos para su diseño. Entre dichos principios se explica cómo hacer uso de aspectos altamente relevantes como la interacción, usabilidad, accesibilidad, realimentación y detección de problemas que pueden estar presentes en estos recursos. Igualmente se discute la importancia de que estos sean intuitivos, atractivos y estimulantes.

Los resultados analizados en pruebas pilotos dan muestra del efecto positivo de todos estos principios en los estudiantes, en especial en lo que se refiere a la motivación. En este aspecto muchos manifestaron que los juegos los incentivaron a esforzarse, a replantear sus modelos mentales sobre varios de los conceptos abordados, logrando así, en definitiva, una mejora en sus habilidades.

\section{Referencias}

BAKER, A., NAVARRO, E., \& VAN DER HOEK, A. An experimental card game for teaching software engineering processes. The Journal of Systems and Software, 75, 3-16. 2005.

BECKER, K. Teaching with games: the minesweeper and asteroids experience. Journal of Computing in Small Colleges, 17(2), 23-33. 2001.

GARRIS, R., AHLERS, R., \& DRISKELL, J. Games, motivation and learning: A research and practice model. Simulation and Gaming, 33(4), 441-467. 2002.

GÓMEZ, P., GONZÁLEZ, P., \& GÓMEZ, M. Aprendizaje basado en juegos. Icono 14, N. 4. 2004.

GORRIZ, C., \& MEDINA, C. Engaging Girls with Computers through Software Games. Communications of the ACM, V. 43, N.1, 42-49. 2000.

HILL, J., RAY, C., BLAIR, J., \& CARVER, C. Puzzles and games: addressing different learning styles in teaching operating systems concepts. In: Proceedings of 34th ACM Special Interest Group on Computer Science Education (SIGCSE), 182-186. 2003.

MOSER, R. A Fantasy Adventure Game as a Learning Environment: Why learning to program is so difficult and what can be done about it. In: Proceedings of the Conference on Integrating Technology in Computer Science Education, 114-116. 1997.

JAMES, J., BEATON, B., CSETE, J., \& VOGEL, D. Mobile educational games. In: Lassner, D., McNaught, C. (Eds.) Proceedings of ED-MEDIA, 801-802. 2003. 
KLOPFER, E., \& YOON, S. Developing Games and Simulations for Today and Tomorrow's Tech Savvy Youth TechTrends. Linking Research \& Practice to Improve Learning, 49(3), 33-41. 2005.

LAWRENCE, R. Teaching data structures using competitive games. IEEE Transactions on Education, 49(1), 459-466. 2006.

LEWANDOWSKI, F., \& SOARES, A. Desenvolvimento de Jogos Educacionais Apoiados por um Agente Tutor Pedagógico. Em: J. Sánchez (Ed.): Nuevas Ideas en Informática Educativa, V.4, 7-14. 2008.

PEREIRA, J., \& RAPKIEWICZ, C. O processo de ensino e aprendizagem de algoritmos e programação: Uma visão crítica da literatura. Em: Workshop de Educacao em Informática de Minas Gerais - WEIMIG. 2002.

PIAGET, J. A epistemología genética. São Paulo, Abril cultural. 1983.

RAPKIEWICKZ, C., FALKEMBACH, G., SEIXAS, L., ROSA, N., DA CUNHA, V., \& KLEMMAN, M. Estratégias pedagógicas no ensino de algoritmos e programação associadas ao uso de jogos educacionais. Revista Novas Tecnologias na Educação, V.4, N. 2. 2006.

RODRIGUES, M. Como ensinar programação. Boletim informativo, V.1, N.1, ULBRA, Canoas. 2002.

ROBERTSON, J., \& HOWELLS, C. Computer game design: Opportunities for successful learning. Computers \& Education, 50, 559-578. 2008.

ZAPATA, C. Teaching software development by means of a classroom game: The software development game. Developments in Business Simulation and Experiential Learning, V. 36, 156-164. 2009. 